\title{
Axisymmetric Vortex Breakdown in a Pipe
}

\author{
S. Wang and Z. Rusak \\ Department of Mechanical Engineering, Aeronautical Engineering and Mechanics \\ Rensselaer Polytechnic Institute, Troy, New York 12180-3590, USA
}

\begin{abstract}
A recent theory of the nearly axisymmetric vortex breakdown in a pipe is presented. We study the motion of an axisymmetric and inviscid swirling flow in a pipe with certain boundary conditions that may reflect the physical situation. The theory is built of global analysis of solutions of the Squire-Long equation and linear stability analysis of these solutions. The theory unifies the major previous theoretical approaches and provides for the first time a consistent explanation of the physical mechanism leading to the vortex breakdown phenomenon as well as the conditions for its occurrence.
\end{abstract}

\section{Introduction}

The breakdown of vortex cores is a remarkable phenomenon in fluid dynamics which refers to the large change in structure of high Reynolds number vortex flows with a high level of swirl. This behavior is still a basic, largely unexplained phenomenon in modern fluid dynamics with a variety of technological applications and scientific interests. Several review papers on this subject have been presented, including $[7,11,13,14,20]$. Those papers show that previous efforts to explain vortex breakdown are based on local analyses and therefore mask important information on the possible development of swirling flows. The study in this paper is motivated by this complicated phenomenon.

This paper concentrates on the nearly-axisymmetric vortex breakdown phenomenon. Experiments of swirling flows in a pipe $[6,8-9,18-20,23]$ provide insight into the structure and stability of the nearly-axisymmetric vortex breakdown. In all of the cases the inlet flow to the pipe, as well as the flow near the inlet decelerating to stagnation and expanding around the nose of the bubble, as well as most of the bubble envelope, show a high degree of axial symmetry [8]. Non axisymmetric perturbations develop mostly near the rear end of the bubble, inside the bubble and downstream in the wake of the bubble and are mostly confined to those regions. The flow inside the recirculation bubble zone is very complicated. Some experiments show that there is an inclined toroidal vortex ring near the downstream end of the recirculation bubble, which gyrates at a regular frequency about the axis of the tube and is accompanied by the lost of axisymmetry near the rear end of the bubble [6, 18-20]. Other experiments [9,23] show the generation of two vortex rings inside the bubble which preserves the axisymmetry of the flow. It can also be observed (see Fig. 8c in [20], Fig. 4 in [5] and Figs. 3, 4 and 6 in [8]) that even downstream of the bubble nose but outside of the bubble and its wake, the flow preserves a degree of axisymmetry. Also, inside the bubble the flow is relatively slow and so the transfer of momentum and kinetic energy from the inside of the bubble to its surroundings is relatively small. The recent experiments of [6] are the only experiments known that describe in details the evolution in time of the breakdown process. They show that the bubble evolves in time with a strong axisymmetry (see Figs. 1 and 5 in [6]). They also show that it is 
only after the axisymmetric bubble has been formed that asymmetric disturbances in the wake of the bubble may only slightly change the flow structure inside the bubble (see Fig. 5 in [6]) but a high degree of symmetry is kept. Bruecker and Althaus [6] also indicated that Sarpkaya [18, 19] had observed a more asymmetric bubble type breakdown than those they found. Therefore, it may be concluded that the non-axisymmetric perturbations observed develop from a base axisymmetric flow that contains a separation zone and to the leading order the bubble evolution and the flow around the bubble is axisymmetric.

As for the viscous effects on the process of vortex breakdown, we can see from the experiments that the nearly axisymmetric bubble develops under certain conditions at about one diameter of the pipe from the inlet. The characteristic time for a particle near the center line to travel from the inlet to the bubble nose is $t=D / U$ ( $D$ is the pipe diameter and $U$ is the averaged axial speed along the centerline). On the other hand the diffusion time over this distance is $t_{d}=D^{2} / \nu$ and for water and air where $\nu$ is small (where the Reynolds number is above 100) it is found that $t<<t_{d}$ so that to the leading order diffusion effects are small and the flow decelerating to stagnation and expanding around the bubble may be considered as inviscid. We recognize, however, that viscous effects become more important on the rear end of the bubble, within the bubble and in its wake, and may create some unsteadiness and determine the bubble position, but the bubble shape and the flow ahead of it are only slightly affected by the small viscosity. Also, the criterion developed by Spall et al. [21] shows no dependence on the Reynolds number $(R e)$ for laminar swirling flows when $R e>100$.

Therefore, the inviscid and axisymmetric framework may serve as a reasonable base frame for the analysis of the nearly-axisymmetric vortex breakdown phenomenon. This framework may give us insight into the mechanisms leading to the creation of the axisymmetric breakdown and the conditions for its appearance. We present in this paper a new theory of the nearly axisymmetric vortex breakdown in a pipe. The theory is built of two major steps: a) Global analysis of the steady state solutions to the Euler equations that describe the motion of an axisymmetric and inviscid swirling flow in a pipe. b) Linear stability analysis of the various steady state solutions that are found. The theory provides for the first time a consistent explanation of the physical mechanism leading to the vortex breakdown phenomenon as well as the conditions for its occurrence. For the detailed theoretical analysis of the phenomenon see the recent papers by Wang and Rusak [24-26] and Rusak et al. [17].

\section{Global analysis of the Squire-Long equation}

We study in this section the solutions to Squire-Long equation (SLE)

$$
\begin{array}{r}
\psi_{r r}-\frac{\psi_{r}}{r}+\psi_{x x}=r^{2} H^{\prime}(\psi)-I^{\prime}(\psi) \\
\text { on } 0 \leq x \leq a, \quad 0 \leq r \leq 1
\end{array}
$$

with boundary conditions

$$
\begin{gathered}
\psi(x, 0)=0, \quad \psi(x, 1)=w_{0} \\
\psi(0, r)=\psi_{0}(r), \quad v(0, r)=\omega v_{0}(r), \quad \psi_{x}(a, r)=0
\end{gathered}
$$

that describe the development of a steady, axisymmetric, incompressible and inviscid swirling flow in a pipe of length $a$. Here, $\psi$ is the stream function, $w=\psi_{r} / r$ is the axial speed $u=-\psi_{x} / r$ is the radial speed, $v$ is the swirl component, $\omega$ is the swirl level and $w_{0}=\psi_{0}(1)$ is the flow flux in the 
pipe. Also, $H$ is the total head function of the flow, $H=p / \rho+\left(u^{2}+w^{2}+v^{2}\right) / 2$, and $I=(r v)^{2} / 2$ is the extended circulation function. For more details on (1) see [1].

The approach presented here is a global variational approach. The analysis is summarized in [24]. Solutions of the SLE are well-known to correspond to the stationary points of the following functional

$$
\mathbf{E}(\psi)=\int_{0}^{a} \int_{0}^{1 / 2}\left(\frac{\psi_{y}^{2}}{2}+\frac{\psi_{x}^{2}}{4 y}+H(\psi)-\frac{I(\psi)}{2 y}\right) d y d x
$$

where $y=r^{2} / 2$. We find that the variational functional $\mathbf{E}(\psi)$ has a rich topological structure (see Fig. 1). Through a careful estimation of the various integrals in (3) we prove the existence of the global minimizer of $\mathbf{E}(\psi)$ when both $H(\psi)$ and $I(\psi)$ are bounded functions, reflecting the boundness of mechanical energy and circulation in the pipe. Then we study the global behavior of the columnar functional $E(\psi)$ (derived from (3) when there is no dependence on $x$ ) and find the various types of stationary points of the columnar functional. We show that when the inlet flow is at a supercritical state it is also a strict local minimizer of $\mathbf{E}(\psi)$. We construct the relations between the properties of the global minimizer of $\mathbf{E}(\psi)$ and the columnar minimizers of $E(\psi)$, i.e., we show that the global minimizer of $\mathbf{E}(\psi)$ is a transition from the given inlet state at $x=0$ to an outlet state at $x=a$ that is the global minimizer of $E(\psi)$ (see Fig. 2). We also show that there exists a special threshold swirl level $\omega_{0}$ where the global minimizer of $\mathbf{E}(\psi)$ changes from a columnar solution all along the pipe when $0<\omega<\omega_{0}$, to a non-columnar solution that describes a swirling flow around an open separation zone when $\omega>\omega_{0}$.

We also rigorously prove the existence of a min-max solution of the SLE (1) using the "MountainPass theorem". The min-max solution exists only above the certain threshold swirl $\omega_{0}$ and its existence is stronly related to the change of nature of the global minimizer solution at $\omega_{0}$ as described above. We study the properties of the min-max solution and show that when the pipe is sufficiently long it may be described by the solitary wave solution of Leibovich and Kribus [15] that bifurcates at the critical level of swirl $\omega_{1}$. For regular situations $\omega_{0}<\omega_{1}$. It can be shown that $\omega_{1}$ tends to the critical level of swirl as defined by Benjamin [2] as the pipe length is increased.

We describe the properties of the above mentioned solutions of the SLE (1) and show that their behavior is different as the swirl of the inlet flow is increased. Finally, we give the complete bifurcation diagram of solutions of the SLE for a supercritical inlet flow, as the swirl is increased from 0 to levels above the critical swirl $\omega_{1}$ (see Fig. 1).

We find that when the swirl level $\omega$ of the inlet flow is less than the threshold value $\omega_{0}$, the inlet flow $\psi_{0}(y)$ develops as a columnar flow all along the pipe and is a global minimizer of $\mathbf{E}(\psi)$ and a unique solution of (1). When $\omega$ is larger than the threshold value $\omega_{0}$ we find for relatively long pipes three possible solutions of the SLE. One is the columnar solution $\psi_{0}(y)$ that develops all along the pipe and is a local minimizer point of $\mathbf{E}(\psi)$. The two other solutions bifurcate at about $\omega_{0}$ from a certain state which is a large disturbance to the columnar flow. One is the global minimizer of $\mathbf{E}(\psi)$ which describes a strong open separation zone (see Fig. 2). The global minimizer solution is the extension of Keller and Egli [12] solution for $\omega>\omega_{0}$. The second solution is the min-max point of $\mathbf{E}(\psi)$ which describes a localized standing wave in the flow (see Fig. 3). The family of min-max points of $\mathbf{E}(\psi)$ also bifurcates at $\omega_{0}$. As the swirl is increased toward $\omega_{1}$ we find that the family of min-max solutions approaches the critical state.

As swirl is increased above the critical level $\omega_{1}$ the columnar flow solution becomes a min-max point of $\mathbf{E}(\psi)$ and a new branch of local minimizer solutions bifurcates at $\omega_{1}$ and may describe a non-columnar flow where the rotating flow is intensified and has a smaller vortical core. The third possible solution when $\omega>\omega_{1}$ is the global minimizer solution described above, where now it can be shown that the separation zone becomes much larger and moves toward the inlet. The results 
also show that the critical swirl is actually a transcritical bifurcation point from which branches of local minimizer solutions and min-max solutions may develop.

\section{$3 \quad$ Stability analyses}

In this section we demonstrate the relation between vortex flow stability and the vortex breakdown phenomenon and specifically the relation between the critical state at the swirl level $\omega_{1}$ and the stability of the vortex flow. We study the linear stability of the various branches of solutions bifurcating at the critical state. From the theory of dynamical systems it is strongly expected that the critical level of swirl is also a point of exchange of stability. The stability analyses are summarized in $[25,26]$.

We first formulate a well posed model of the unsteady motion of swirling flows with compatible boundary conditions which are similar to those used in the steady analysis.

An unsteady, axisymmetric, incompressible and inviscid flow with swirl is considered in a finite length pipe of a unit radius, the centerline of which is the $x$-axis and where $0 \leq x \leq a$. By virtue of the axisymmetry, a stream function $\psi(x, y, t)$ can be defined where the radial component of velocity $u=-\psi_{x} / \sqrt{2 y}$, and the axial component of velocity $w=\psi_{y}$. The azimuthal vorticity $\eta$ is given by $\eta=\sqrt{2 y} \chi$ where $\chi=-\left(\psi_{y y}+\psi_{x x} / 2 y\right)$. The circulation function $K$ is defined as $K=\sqrt{2 y} v$ where $v$ is the circumferential velocity. The equations which connect the development in time $(t)$ of the stream function $\psi$, the function $\chi$ and the circulation function $K$ may be given by (see for example $[22])$

$$
\begin{array}{r}
K_{t}+\{\psi, K\}=0, \\
\chi_{t}+\{\psi, \chi\}=\frac{1}{4 y^{2}}\left(K^{2}\right)_{x} .
\end{array}
$$

Here the brackets $\{\psi, K\}$ and $\{\psi, \chi\}$ are defined by

$$
\begin{array}{r}
\{\psi, K\}=\psi_{y} K_{x}-\psi_{x} K_{y}, \\
\{\psi, \chi\}=\psi_{y} \chi_{x}-\psi_{x} \chi_{y}
\end{array}
$$

We study the development of the flow in the pipe with certain conditions posed on the boundaries. For any time $t$ we set $\psi(x, 0, t)=0$ to satisfy the axisymmetric condition along the pipe centerline, and $\psi(x, 1 / 2, t)=w_{0}$ to describe the total mass flux across the pipe. Also, let for any time $t$

$$
\psi(0, y, t)=\psi_{0}(\sqrt{2 y}), \quad K(0, y, t)=\omega \sqrt{2 y} v_{0}(\sqrt{2 y})
$$

be given along the inlet. We also consider the case where $\psi_{x x}(0, y, t)=0$ to fix the azimuthal vorticity $\chi$ along the inlet at any time $t$,

$$
\chi(0, y, t)=-\psi_{0 y y} .
$$

The boundary conditions along the outlet depend on the physical situation. However, boundary conditions that result in a well posed mathematical problem may relate to certain physical situations of interest. The method given in this paper is general and can handle various conditions, but in order to demonstrate our idea we concentrate on specific outlet conditions. According to the theory of partial differential equations we need to specify only one condition along the pipe outlet. We assume no radial speed along $x=a$

$$
\psi_{x}(a, y, t)=0
$$


We consider now a base, steady and non-columnar swirling flow where $\psi=\psi_{0}(x, y)$ and $K=$ $K_{0}(x, y)$. This base flow is a steady state solution of (4) through (9) for which $K_{0}=K_{0}\left(\psi_{0}\right)$ and $\psi_{0}(x, y)$ is a solution of the Squire-Long equation.

To study the stability of this base flow we let

$$
\begin{aligned}
& \psi(x, y, t)=\psi_{0}(x, y)+\epsilon \psi_{1}(x, y, t)+\ldots \\
& K(x, y, t)=K_{0}(x, y)+\epsilon K_{1}(x, y, t)+\ldots
\end{aligned}
$$

where $\epsilon \psi_{1}$ is the disturbance to the stream function and $\epsilon K_{1}$ is the circulation disturbance. On substituting these expressions into (5) and neglecting second order terms, we then obtain the linearized equations of motion of the swirling flow

$$
\begin{array}{r}
K_{1 t}+\psi_{0 y} K_{1 x}-K_{0 y} \psi_{1 x}+K_{0 x} \psi_{1 y}-\psi_{0 x} K_{1 y}=0 \\
\chi_{1 t}+\psi_{0 y} \chi_{1 x}-\chi_{0 y} \psi_{1 x}+\chi_{0 x} \psi_{1 y}-\psi_{0 x} \chi_{1 y}=\frac{\left(K_{0} K_{1}\right)_{x}}{2 y^{2}} .
\end{array}
$$

Here $\chi_{1}=-\left(\psi_{1 y y}+\psi_{1 x x} / 2 y\right)$ is related to the disturbance of the azimuthal vorticity.

From the above equations we may get

$$
\int_{0}^{x} \mathbf{F}\left(\psi_{1}\right)_{t} d x+\psi_{0 y} \mathbf{F}\left(\psi_{1}\right)-\frac{\psi_{0 x}}{y^{2}}\left[y^{2} \int_{0}^{x} \mathbf{F}\left(\psi_{1}\right) d x\right]_{y}+\frac{I^{\prime}\left(\psi_{0}\right)}{2 y^{2}} \psi_{1 t}=0
$$

where

$$
\mathbf{F}\left(\psi_{1}\right)=\chi_{1 t}-\psi_{0 y}\left(\mathbf{L}\left(\psi_{1}\right)\right)_{x}+\psi_{0 x}\left(\mathbf{L}\left(\psi_{1}\right)\right)_{y} .
$$

and where

$$
\mathbf{L}\left(\psi_{1}\right)=\psi_{1 y y}+\frac{\psi_{1 x x}}{2 y}-\left(H^{\prime \prime}\left(\psi_{0}\right)-\frac{I^{\prime \prime}\left(\psi_{0}\right)}{2 y}\right) \psi_{1}
$$

is the linearized operator of the Squire-Long equation (1).

We consider now a suitable mode analysis of (12) of the form

$$
\psi_{1}=\phi(x, y) e^{\sigma t}
$$

where in the general case $\sigma$ may be a complex number and $\phi(x, y)$ and $k(x, y)$ are complex functions.

Using asymptotic techniques in the limit $\omega$ tends to $\omega_{1}$ and $\sigma$ tends to zero we find asymptotic solutions to the linearized equations and show that the transcritical point of bifurcation at $\omega_{1}$ is also a point of exchange of stability of the branches of the columnar solutions and the non-columnar solutions bifurcating at $\omega_{1}[25,26]$. Results are summarized in Fig. 4. We can see that the branches of local minimizer solutions have an asymptotically stable mode of disturbance whereas the branches of min-max solutions are linearly unstable. Specifically, the branch of columnar flow all along the pipe is asymptotically stable for $\omega<\omega_{1}$ and becomes unstable when $\omega>\omega_{1}$.

The results presented in [25] also provide an interesting physical picture, specifically for the stability of columnar swirling flows. When there is no swirl, the flow cannot sustain any disturbance and any initial disturbance is convected downstream by the axial flow and is swept out from the flow domain in a finite time. When swirl is introduced, disturbances to the flow propagate at different speeds that depend on the swirl level [2]. There exists the critical level of swirl $\omega_{1}$ where the largest speed of the disturbances propagating upstream is equal to the axial speed of the base flow and then the flow can sustain a neutrally stable standing wave with a finite length. This mode of disturbance 
is stable at a supercritical state $\left(\omega<\omega_{1}\right)$ because less swirl results in a smaller value of the largest speed of the disturbances propagating upstream and, therefore, the washout effect of the axial flow is more dominant, resulting in the decay of the disturbance in time. However, when the swirl is greater than the critical level, the disturbance mode is unstable since higher swirl results in higher values of the largest speed of the disturbances propagating upstream and, therefore, they become more dominant. Since the inlet conditions (7) and (8) for the axial and circumferential velocity components and the azimuthal vorticity are fixed for all time the disturbance moving upstream can not propagate through the inlet and also cannot be reflected from the inlet and, therefore, tends to accumulate and create an instability.

\section{Mechanism of the axisymmetric vortex breakdown in a pipe}

The above results shed light on the physical mechanism leading to the axisymmetric vortex breakdown phenomenon in a pipe. As the swirl along the inlet of the pipe is increased toward the critical level, the base columnar vortex flow tends to lose its stability margin and definitely above the critical level it is unstable (see Fig. 4). Then, near or above the critical swirl $\omega_{1}$, any finite perturbation will induce a transition from the columnar state to another possible state. It is clear that the min-max solution is unstable and, therefore, is not a final steady state. Moreover, the only other steady state that is possible is the global minimizer solution of the Squire-Long equation. As discussed above, the global minimizer solution exists and above a certain threshold level of swirl $\omega_{0}$, it describes a swirling flow with a large separation zone that resembles the breakdown zone. Our recent numerical simulations demonstrate that the global minimizer solution when $\omega>\omega_{0}$ is stable to axisymmetric disturbances [17]. In this way, the mechanism of the axisymmetric vortex breakdown phenomenon is explained as a transition, that may occur only when $\omega>\omega_{0}$, and definitely when $\omega>\omega_{1}$, from a columnar vortex flow that loses its stability to another completely different steady and stable state which is the global minimizer solution for the same boundary conditions and that can be thought as a strong attractor.

We can find estimates for $\omega_{0}$ and $\omega_{1}$ as functions of the core radius $r_{0}$ in Keller and Egli [12], Fig. 11 where the inlet flow is modeled by the Rankine vortex and the separation region is modeled by a stagnation zone. Specifically, when the vortex core is very slender ( $r_{0}$ is small) they found that $2 \omega_{0} r_{0} / w_{0}=\sqrt{2}$ and $2 \omega_{1} r_{0} / w_{0}=2.405$. Here $w_{0}$ is the axial speed in the center of the vortex core. These results may also be good estimates for $\omega_{0}$ and $\omega_{1}$ of a free concentrated vortex, that can be modeled by the limit case where the pipe radius tends to infinity. When $r_{0} / R(R$ is the pipe radius) increases the two swirl ratios $2 \omega_{0} r_{0} / w_{0}$ and $2 \omega_{1} r_{0} / w_{0}$ also increase but get closer and merge when $r_{0} / R=1$ (a complete solid body rotation in the pipe) at a value of 3.832 . When the inlet flow is given by a different model such as the Burgers' vortex our recent computations provide the breakdown criteria as function of the core radius of the vortex [17]. Results show similar behavior to those of the Rankine vortex. Estimations of the swirl ratio $2 \omega r_{0} / w_{0}$ from the experimental data of Garg and Leibovich [10] and Uchida et al. [23] also show that breakdown occurs when $\omega$ is above $\omega_{0}$.

\section{Conclusions}

The theory presented in this paper provides a consistent explanation of the mechanism of the axisymmetric vortex breakdown phenomenon. It is a transition from a columnar vortex flow that tends to lose its stability to another steady and stable state which is contains a large separation zone. The theory explains the suddenness of the vortex breakdown phenomenon, specifically around 
$\omega_{0}$ and where a large disturbance to the base flow occurs. The theory also establishes the relation between the critical swirl $\omega_{1}$ and the stability of the flow in the pipe. The theory predicts the nonuniqueness of the phenomena related with the process of increasing the swirl from $\omega_{0}$ to $\omega_{1}$. All of these characteristics are similar to those found in the experiments of Sarpkaya [18, 20], Leibovich $[13,14]$ and Bruecker and Althaus [6] where nearly axisymmetric bubbles were found. The numerical computations of vortex breakdown of Beran and Culick [4] show bifurcation diagrams of solutions that also depend on the swirl (see their Figs. 6, 7 and 13) and agree with our bifurcation diagram. Also, the time dependent calculations of Beran [3] and Lopez [16] agree with the stability results.

The present theory also unifies most of the previously suggested approaches to axisymmetric vortex breakdown into a global theory and provides guidelines for future numerical simulations and experiments of this complicated phenomenon.

\section{Acknowledgement}

This research was carried out with the support of the National Science Foundation under Grant CTS-9310181, monitored by Dr. R. Powell and Dr. D.S. Ahluwalia.

\section{References}

[1] Batchelor, G.K., An Introduction to Fluid Dynamics, Cambridge University Press, 1967.

[2] Benjamin, T.B., "Theory of the Vortex Breakdown Phenomenon," J. Fluid Mech., 14, pp. 593-629, 1962.

[3] Beran, P.S., "The Time-Asymptotic Behavior of Vortex Breakdown in Tubes", Comp. Fluids, 23 (7), pp. 913-937, 1994.

[4] Beran, P.S. and Culick, F.E.C., "The Role of Non-Uniqueness in the Development of Vortex Breakdown in Tubes", J. Fluid Mech., 242, pp. 491-527, 1992.

[5] Bruecker, C. and Althaus, W., "Study of Vortex Breakdown by Particle Tracking Velocimetry (PTV), Part 1: Bubble-type Vortex Breakdown," Exp. Fluids, 13, pp. 339-349, 1992.

[6] Bruecker, C. and Althaus, W., "Study of Vortex Breakdown by Particle Tracking Velocimetry (PTV), Part 3: Time-Dependent Structure and Development of breakdown Modes," Exp. Fluids, 18, pp. 174-186, 1995.

[7] Escudier, M.P., "Vortex Breakdown: Observations and Explanations," Prog. Aerospace Sciences, 25, pp. 189-229, 1988.

[8] Faler, J.H. and Leibovich, S., "Disrupted States of Vortex Flow and Vortex Breakdown," Phys. Fluids, 20, pp. 1385-1400, 1977.

[9] Faler, J.H. and Leibovich, S., "An Experimental Map of the Internal Structure of a Vortex Breakdown," J. Fluid Mech., 86 (2), pp. 313-335, 1978.

[10] Garg, A.K. and Leibovich, S., "Spectral Characteristics of Vortex Breakdown," Phys. Fluids, 22 (11), pp. 2053-2064, 1979.

[11] Hall, M.G., "Vortex Breakdown," Ann. Rev. Fluid Mech., 4, pp. 195-217, 1972. 
[12] Keller, J.J. and Egli, W., "Force- and Loss-Free Transitions Between Flow States," J. App. Math. and Phys. (ZAMP), 36, pp. 854-889, 1985.

[13] Leibovich, S., "The Structure of Vortex Breakdown," Ann. Rev. Fluid Mech., 10, pp. 221-246, 1978.

[14] Leibovich, S., "Vortex Stability and Breakdown: Survey and Extension," AIAA J., 22, pp. 1192-1206, 1984.

[15] Leibovich, S. and Kribus, A., "Large Amplitude Wavetrains and Solitary Waves in Vortices," J. Fluid Mech., 216, pp. 459-504, 1990.

[16] Lopez, J.M., "On the Bifurcation Structure of Axisymmetric Vortex Breakdown in a Constricted Pipe," Phys. Fluids, 6 (11), pp. 3683-3693, 1994.

[17] Rusak, Z., Wang, S. and Whiting, C., "Numerical Computations of Axisymmetric Vortex Breakdown in a Pipe," AIAA Paper 96-0801 (submitted to J. Fluid Mech.), 1996.

[18] Sarpkaya, T., "On Stationary and Traveling Vortex Breakdown," J. Fluid Mech., 45 (3), pp. 549-559, 1971.

[19] Sarpkaya, T., "Effect of Adverse Pressure Gradient on Vortex Breakdown," AIAA J., 12 (5), pp. 602-607, 1974.

[20] Sarpkaya, T., "Vortex Breakdown and Turbulence," AIAA paper 95-0433, 1995.

[21] Spall, R.E., Gatski, T.B. and Grosch, C.E., "A Criterion for Vortex Breakdown," Phys. Fluids, 30, pp. 3434-3440, 1987.

[22] Szeri, A. and Holmes, P., "Nonlinear Stability of Axisymmetric Swirling Flows," Phil. Trans. Roy. Soc. Lond., A326, pp. 327-354, 1988.

[23] Uchida, S., Nakamura, Y. and Ohsawa, M., "Experiments on the Axisymmetric Vortex Breakdown in a Swirling Air Flow," Tran. Japan Soc. of Aeron. and Space Sciences, 27 (78), pp. 206-216, 1985.

[24] Wang, S. and Rusak, Z., "A Theory of the Axisymmetric Vortex Breakdown in a Pipe," R.P.I. Aeron. Eng. Report 107 (submitted to J. Fluid Mech.), 1995.

[25] Wang, S. and Rusak, Z., "On the Stability of an Axisymmetric Rotating Flow in a Pipe," Phys. Fluids, 8 (4), pp. 1007-1016, 1996.

[26] Wang, S. and Rusak, Z., "On the Stability of Non-Columnar Swirling Flows", Phys. Fluids, 8 (4), pp. 1017-1023, 1996. 


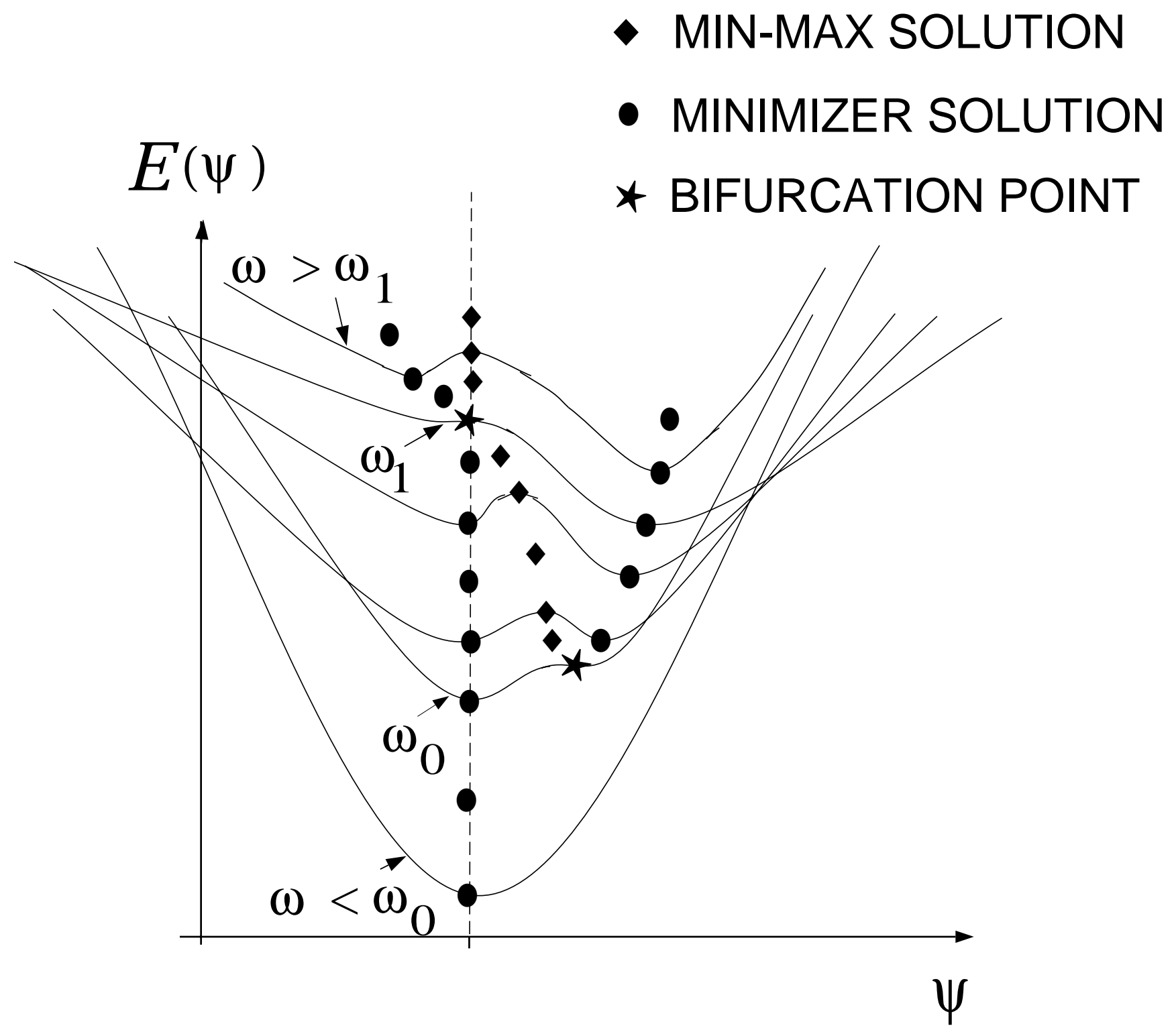

Figure 1: The bifurcation diagram of solutions to the Squire-Long equation. 


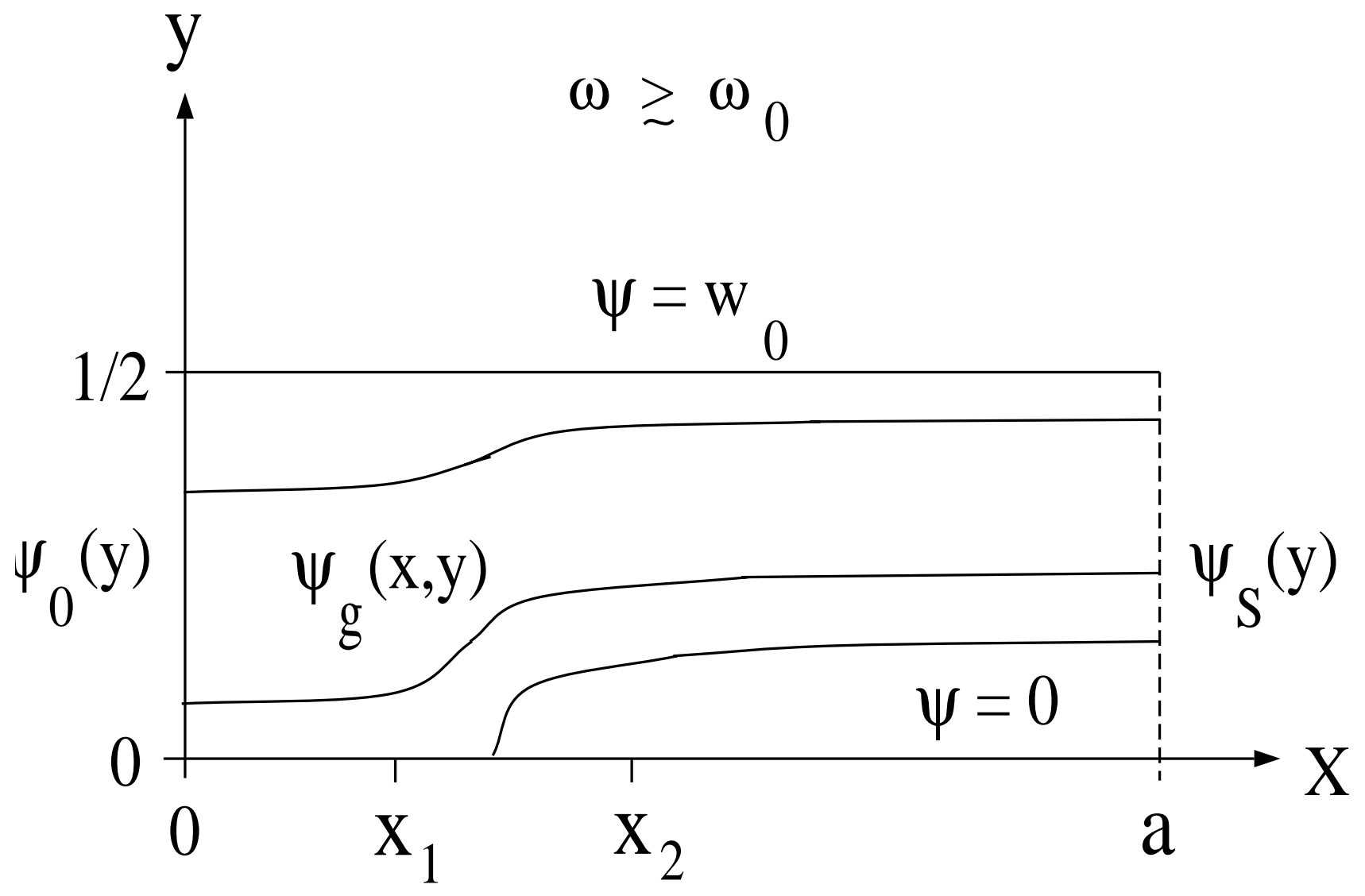

Figure 2: The global minimizer solution $\psi_{g}(x, y)$ when $\omega \geq \omega_{0}$. 


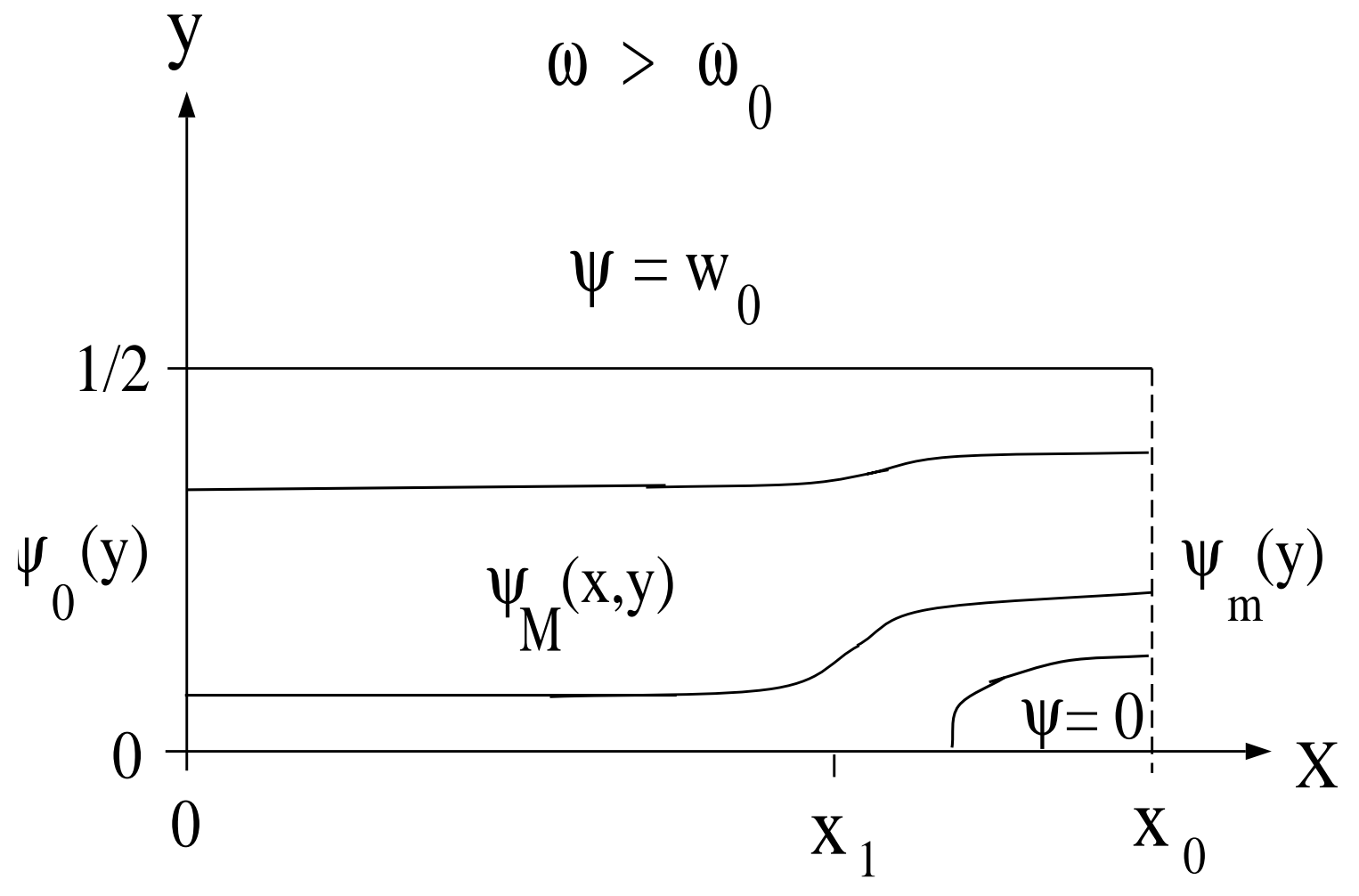

Figure 3: The minmax solution when $\omega_{0}<\omega<\omega_{1}$. 


\section{STABLE SOLUTION \\ UNSTABLE SOLUTION \\ * BIFURCATION POINT}

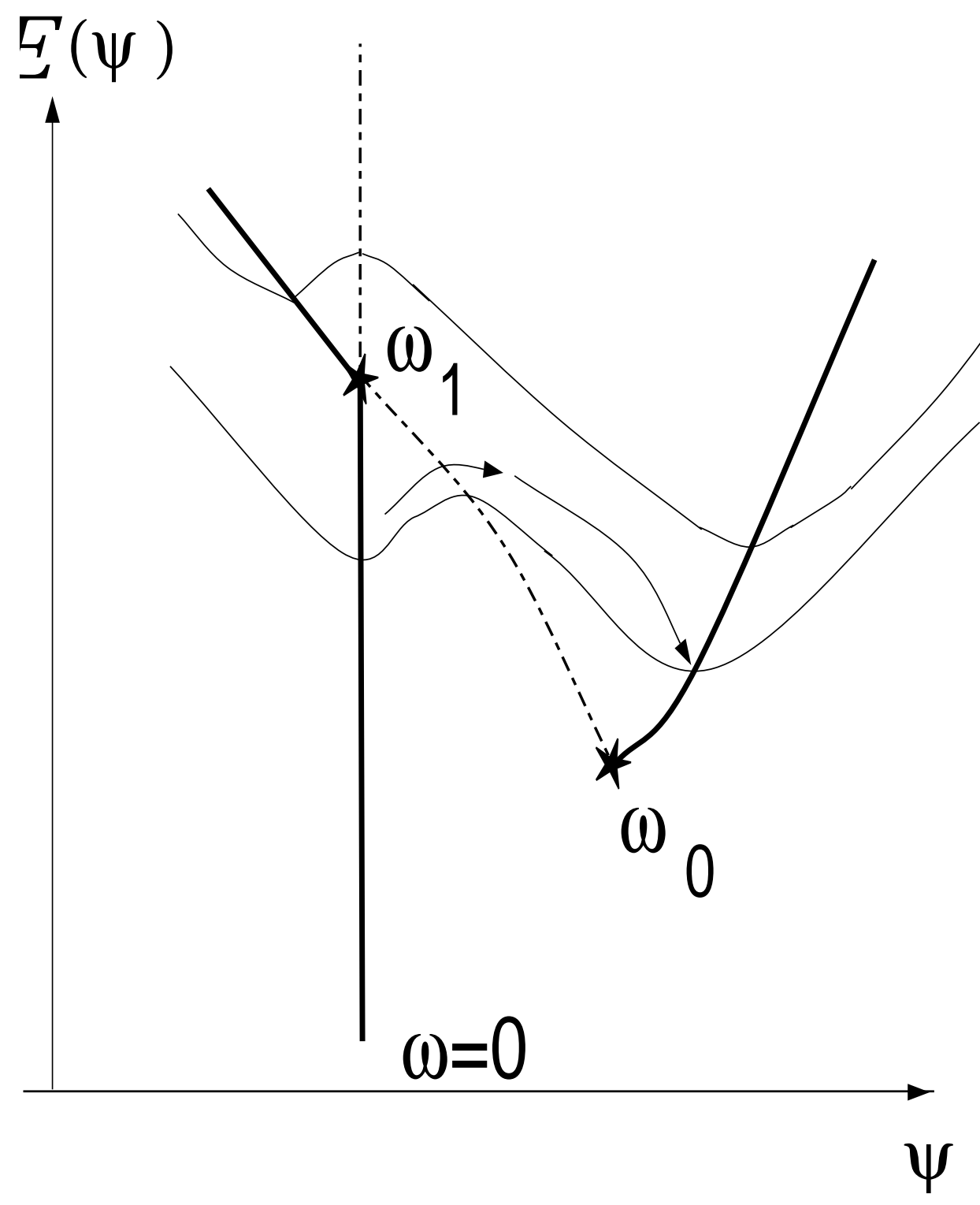

Figure 4: The bifurcation diagram and stability characteristics. 